\title{
Aeroelastic Effects in Maximum Lift Prediction of a Transport Aircraft and Comparison to Flight Data
}

\author{
Stefan Keye* and David Rohlmann ${ }^{\dagger}$ \\ DLR, German Aerospace Center, \\ Institute of Aerodynamics and Flow Technology, 38108 Braunschweig, Germany
}

\begin{abstract}
The virtual determination of maximum lift performance of a transport aircraft configuration taking into account static aeroelastic deformations at steady-state flight conditions is described. Within the scope of the national research project HINVA (High-Lift INFlight VAlidation) a fluid-structure interaction approach based on high-fide $\overline{\overline{i t} y}$ numerical fluid dynamics and structural analysis methods has been applied to DLR's Airbus A320232 ATRA research aircraft in high-lift configuration. Numerical aeroelastic analyses were performed using an in-house simulation procedure built around DLR's flow solver TAU and the commercial finite-element analysis code NASTRAN ${ }^{\circledR}$. Numerical results were validated against experimental data obtained from the first HINVA flight test campaign in July 2012.
\end{abstract}

\section{Introduction}

7 HE accurate numerical prediction of maximum achievable lift and the corresponding angle of attack of 1 a transport aircraft configuration are of particular importance for the overall design and the reduction of financial risks inherent to the development of new aircraft configurations. Against this background, the national research project HINVA $^{1}$ has been established within the framework of the fourth German Aeronautical Research Program LuFo IV. HINVA's main goal is the reliable and accurate prediction of maximum lift for a representative transport aircraft configuration, Figure 1, using numerical simulation methods and cryogenic wind tunnel testing. The prediction accuracy of both numerical and experimental simulation approaches with respect to flight test data targeted in HINVA is $\Delta C_{L, \max } \leq \pm 2 \%( \pm 0.05)$ for maximum lift and $\Delta \alpha\left(C_{L, \max }\right) \leq \pm 2 \%( \pm 0.4 \mathrm{deg})$ for the associated angle of attack. A physically correct simulation of all aerodynamic effects relevant for maximum lift is essential for:

- the trustworthiness of simulation results in general,

- the identification and quantification of sources of deviations between computational fluid dynamics (CFD) simulations, wind tunnel experiments, and flight test,

- the improvement of flight physics knowledge for high-lift configurations, and

- the derivation of conclusions regarding the specific future development of numerical methods.

Work presented in this paper focuses on the assessment of static aeroelastic deformations of the wing, high-lift devices, fuselage, and empennage in the context of high-lift aerodynamics and flow separation behavior. Results are compared to numerical data obtained from conventional CFD analyses and validated against flight test data.

\footnotetext{
* Research Scientist, Dept. Transport Aircraft.

${ }^{\dagger}$ Research Scientist, Dept. Transport Aircraft.
} 


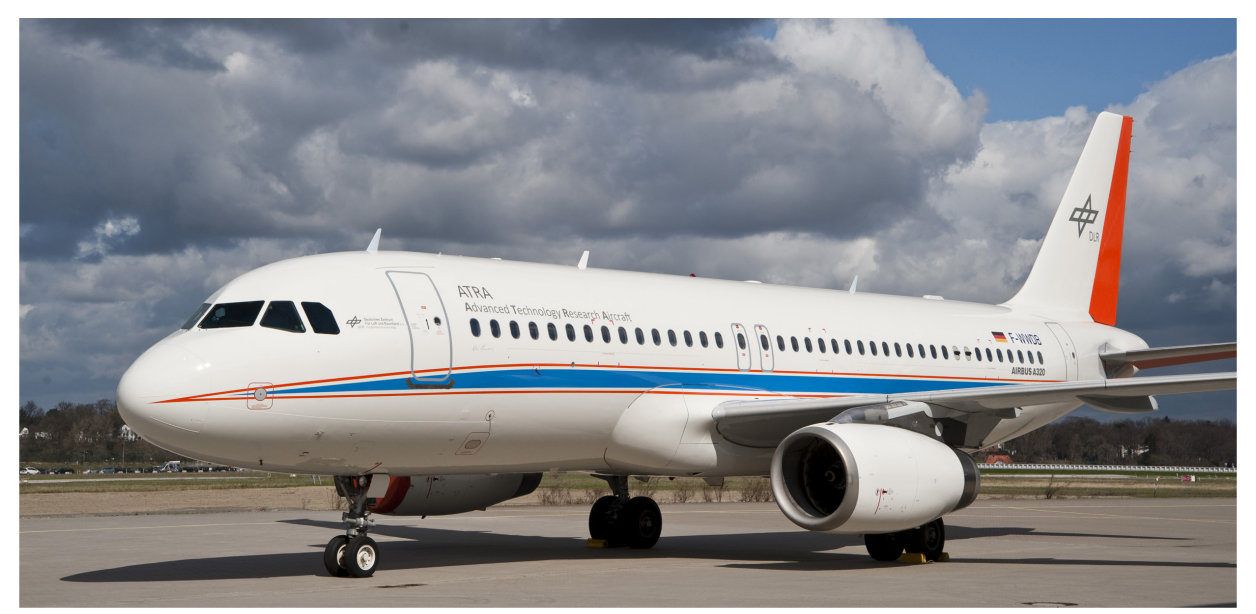

Figure 1. DLR's Airbus A320-232 Advanced Technology Research Aircraft (ATRA).

\section{Determination of Static Equilibrium}

DLR's fluid-structure coupled (FSC) simulation procedure, Fig. 2, is based on a direct coupling of highfidelity CFD and computational structural mechanics (CSM) methods. ${ }^{2}$ The simultaneous interaction of outer flow field and flexible aircraft structure is modeled through alternately solving the Reynolds-averaged Navier-Stokes equations and the basic equations of structural mechanics, and the interpolation of aerodynamic forces and structural deflections over the common surface of CFD and structural grids. For the investigations described here, DLR's in-house flow solver $\mathrm{TAU}^{3}$ and the commercially available structural analysis code NASTRAN ${ }^{\circledR 4}$ were used.

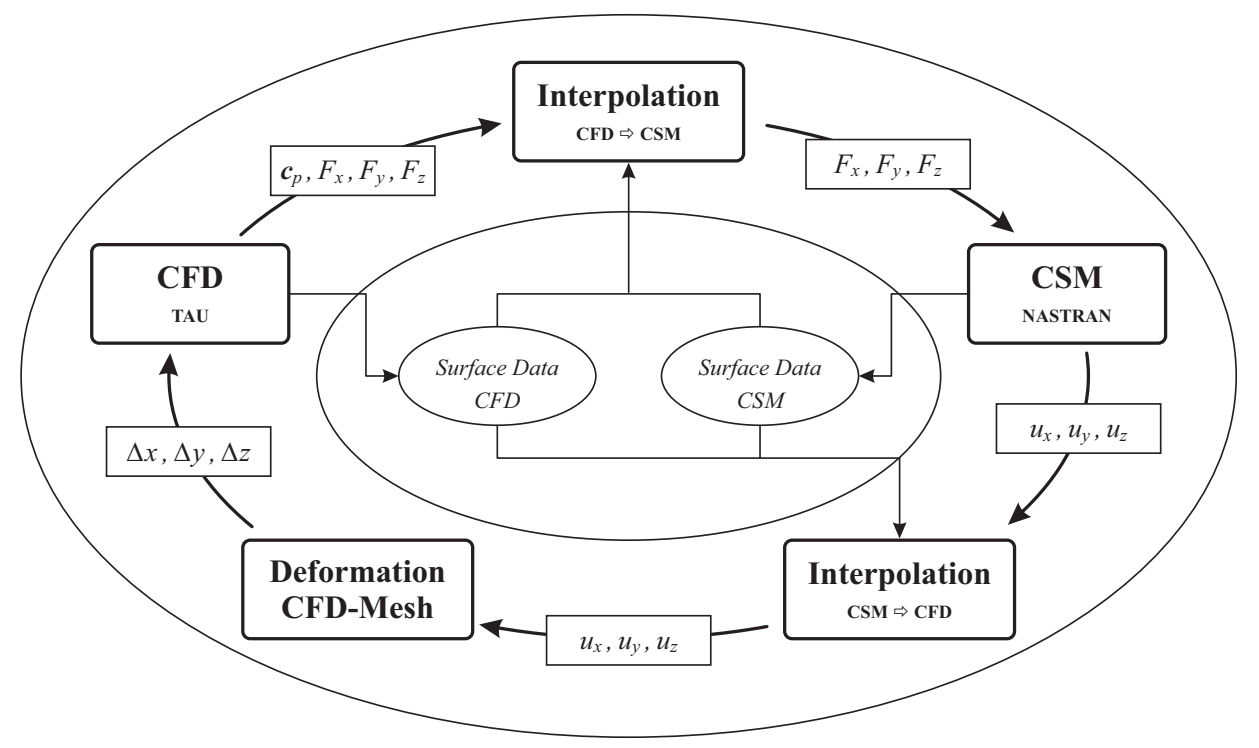

Figure 2. Numerical simulation procedure for aero-elastic analyses.

\section{Numerical Models and Simulations}

\section{A. CFD Mesh}

The geometric model used in HINVA is a highly realistic and detailed representation of DLR's A320-232 ATRA research aircraft in landing configuration. In order to enable a most accurate numerical predic- 
tion of maximum lift, only minor simplifications, inevitable for successful mesh generation, were introduced. $^{5}$ The unstructured, hybrid CFD mesh was generated using the commercial grid generation software CENTAUR $^{\mathrm{TM}}{ }^{6}$ Particular attention was dedicated to those geometric features most relevant for the development of the inboard wing vortex system which essentially determines overall high-lift performance. These include slat tracks, de-icing pipe, slat horn, and nacelle strake. For each test case the horizontal tail plane (HTP) and elevator angles were set to the values recorded in the flight log. To enable the use of a single grid for all CFD computations, the prismatic boundary layer elements were designed such that a non-dimensional first spacing of $y^{+} \leq 1$ and a final prism height of approximately twice the maximum boundary layer thickness is ensured for the entire range of flight test flow conditions. The final CFD mesh, Figure 3, has $80.3 \times 10^{6}$ grid points.

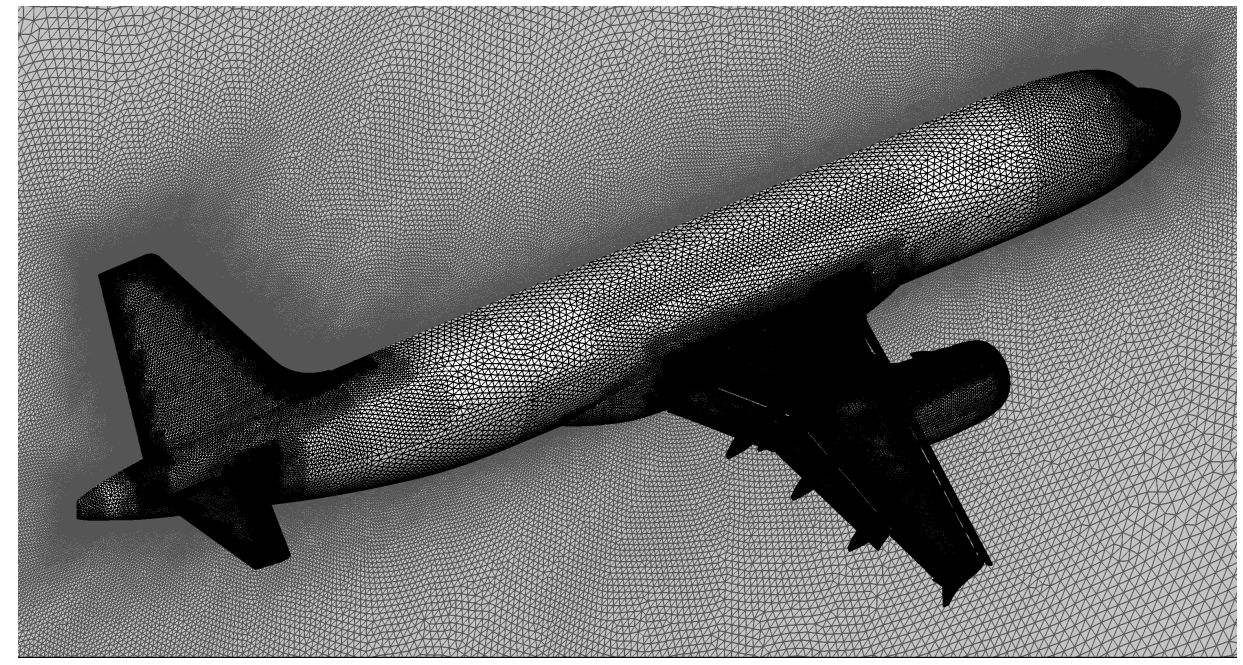

Figure 3. A320-232 CFD surface (black) and symmetry plane (gray) meshes.

All CFD results shown here were performed using a central spatial discretization scheme with Jamesontype artificial dissipation in matrix mode, ${ }^{7,8}$ an implicit LU-SGS time integration scheme, a ' $3 \mathrm{v}$ ' multi-grid cycle, and the one-equation Spalart-Allmaras turbulence model. ${ }^{9}$

\section{B. Structural Model}

A NASTRAN ${ }^{\circledR}$ finite-element model of wing box, fuselage, horizontal stabilizer, vertical tailplane, and engines was made available by AIRBUS Operations GmbH, Figure 4. Contrary to the CFD mesh, a full model is used to take into account structural asymmetries, like cabin and cargo doors or secondary mass distribution. The model consists of 25,200 nodes and 58,900 elements and has 151,000 degrees of freedom. A modal validation was performed using ground vibration test data. Subsequent model corrections were restricted to an adaptation of the stiffness distribution in order to ensure accurate results for both dynamic and static analyses. High-lift devices, i.e. slats, slat tracks, inner and outer flaps, and flap tracks, are represented by Timoshenko beam elements. Fuel tanks have been added to enable the correct modeling of the amount and distribution of fuel mass.

All simulations were performed using NASTRAN ${ }^{\circledR}$ 's linear, static solution sequence 101 . For the inflight analysis cases a 'free-free' suspension was constituted based on the 'inertia relief' option. ${ }^{10}$ Fuel mass was individually adjusted to the existing amount of fuel at the point of data acquisition. Engine thrust values were derived from the computed drag coefficient and introduced as discrete structural forces, equally distributed onto the engine pivot nodes. Ground shapes (cf. Chapter IV, Section B) were determined by suspending the aircraft on the main and nose landing gear and subjecting it to a $1 \mathrm{~g}$ gravitational load.

\section{Coupling of Aerodynamic Loads and Structural Deflections}

In previous applications, coupling of aerodynamic loads and structural deflections between CFD simulation and finite-element analysis was implemented on the main wing and high-lift system only. To study the aerodynamic influence of body and empennage deformations on maximum lift prediction, the existing coupling 


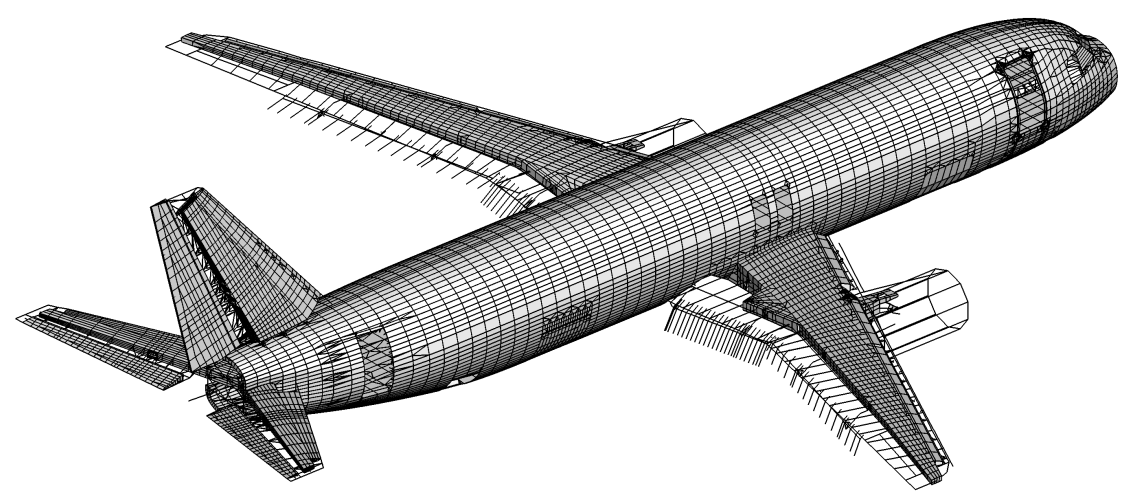

Figure 4. A320-232 finite-element model.

approach has been extended to fuselage, horizontal stabilizer and vertical tailplane. This was assumed to allow for a more realistic simulation of the lift and pitching moment contributions of horizontal stabilizer and fuselage to overall lift and trim state and therefore enable a more accurate determination of aerodynamic loads acting on the wing.

With high-lift configurations featuring multiple aerodynamic surfaces a component-based interpolation procedure for both aerodynamic forces and structural deflections is used. This ensures the correct allocation of forces to the individual high-lift elements and accounts for rigid body deflections of flaps and slats relative to the wing. For the A320-232 ATRA the components are (1) wing, (2) inboard and outboard flap, (3) inboard slat, and (4) outboard slats.

\section{Comparison of numerical Results and Flight Test Data}

\section{A. Test Vehicle}

The test carrier used for the HINVA flight test campaign is DLR's Airbus A320-232 D-ATRA. The aircraft has a length of $37.57 \mathrm{~m}(123.3 \mathrm{ft})$, a wing span of $34.10 \mathrm{~m}(111.9 \mathrm{ft})$, and the aerodynamic mean chord is $4.19 \mathrm{~m}$ (13.76ft). Empty weight is 42.3 to $(93,255 \mathrm{lb})$ and the maximum takeoff weight is 75.5 to $(166,450 \mathrm{lb})$. The aircraft performed its first flight on January 24, 1997 and has joined DLR's fleet of research aircraft in 2008.

\section{B. Instrumentation}

During the first HINVA flight test campaign in July 2012 the A320-232 ATRA was equipped with numerous measurement techniques, Figure 5, including:

- static pressure belts for high resolution chordwise pressure distributions of wing and HTP,

- Kulite sensors for dynamic pressure measurements in separation regions and wake areas,

- flow cones and video cameras for flow separation observations,

- hot film arrays for transition detection,

- stereo still cameras, checkerboard markers, and random dot patterns for deformation measurements on wing and high-lift devices.

For validation of CFD and FSC simulations, only chordwise static pressure distributions from both the wing and HTP pressure belts and wing deformation data from the checkerboard markers were used, Figure 6. Wing pressure belts were located in five spanwise sections at $\eta=0.15,0.25,0.40,0.62$, and 0.84 . Slat pressure distributions are available from sections no. 2 to no. 5 , whereas sections no. 1 to no. 4 include flap pressure distributions. Due to technical constraints the upper wing belt segment of section no. 3 had to be shifted 


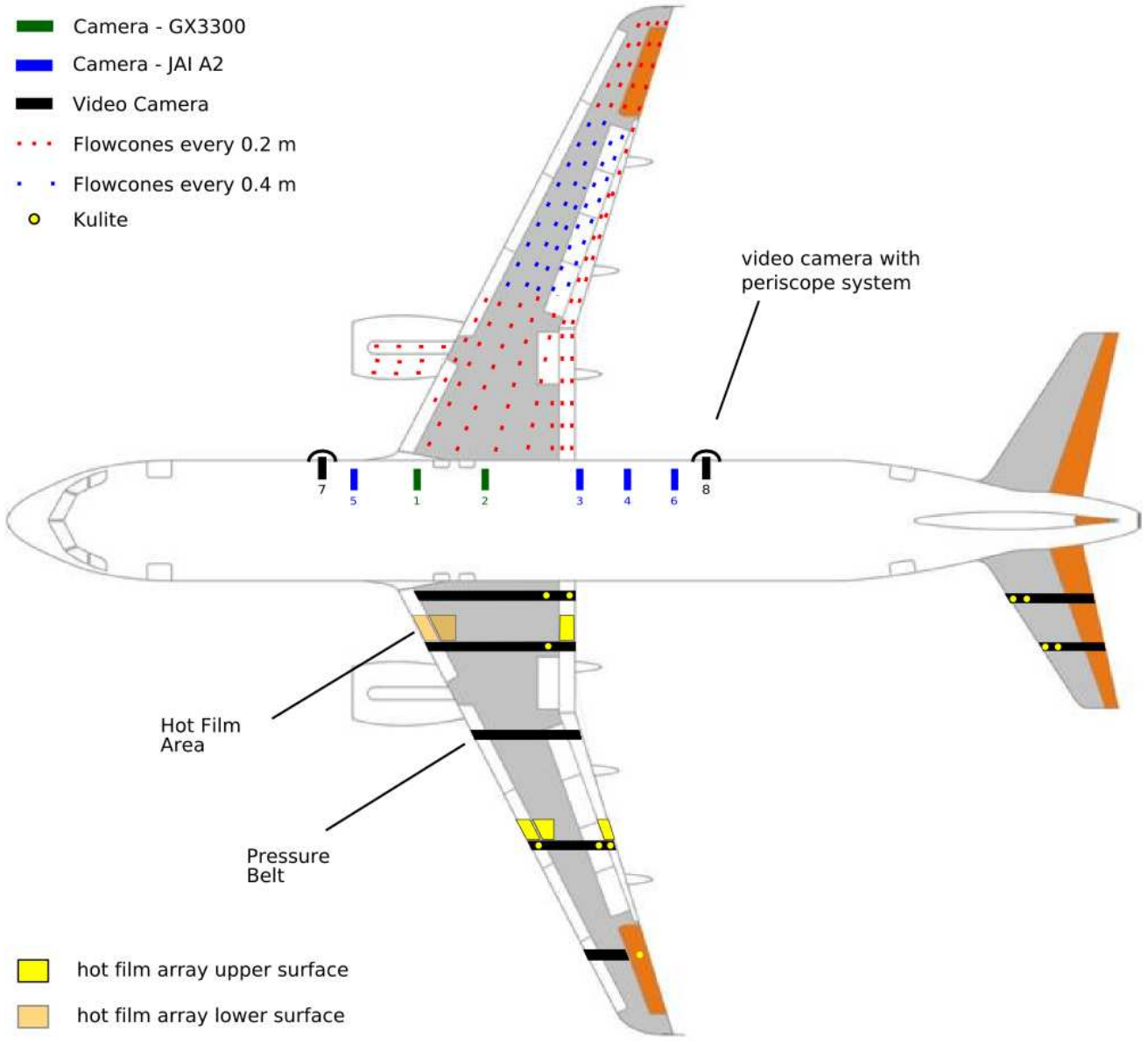

Figure 5. A320-232 ATRA instrumentation during first HINVA flight test campaign (deformation markers and dot pattern areas not shown).

inboard by $21.4 \mathrm{~cm} / 8.43 \mathrm{in}$. Pressure belts on the HTP were located at $\eta=0.45$, and 0.675 . The total number of pressure taps was 348 for the wing and 58 for the HTP.

Wing bending and twist deformations were measured using 38 checkerboard markers in 10 spanwise sections between $\eta=0.350$, and 0.952 and four markers on the tip fence. Deflections were computed from the differences of marker coordinates between the in-flight state and a reference state while the aircraft was on the ground. Hence, all measured deformations represent relative deformations between the flight shape and the reference ground shape. Computed deflections, in contrast, are related to the undeformed jig shape geometry, preventing a direct comparison to measured results. Therefore, an additional structural analysis is required to determine a numerical ground shape (cf. Chapter III, Section B) and enable a comparison of numerical and experimental deformation data. Accordingly, all experimental and numerical deformation data presented in this chapter will be plotted as difference between flight and ground shape, i.e., $\Delta w=w_{\text {Flight }}-w_{\text {Ground }}$ and $\Delta \varepsilon=\varepsilon_{\text {Flight }}-\varepsilon_{\text {Ground }}$.

\section{Test Cases}

Two low-speed test cases, Table 1, with high-lift devices set to the 'FULL' or landing position were selected for comparison of numerical simulations and flight test data. Owing to HINVA's focus on maximum lift prediction and in order to avoid any dynamic effects during stall maneuvers, data from stabilized points ${ }^{\mathrm{a}}$ at high angles of attack close to maximum lift will be used. It should be noted that due to technical constraints it was not possible to use all measurement techniques installed during the first flight test campaign

\footnotetext{
${ }^{\mathrm{a}}$ In a stabilized point data is acquired while the aircraft is in level flight at constant speed and angle of attack over a fixed period.
} 


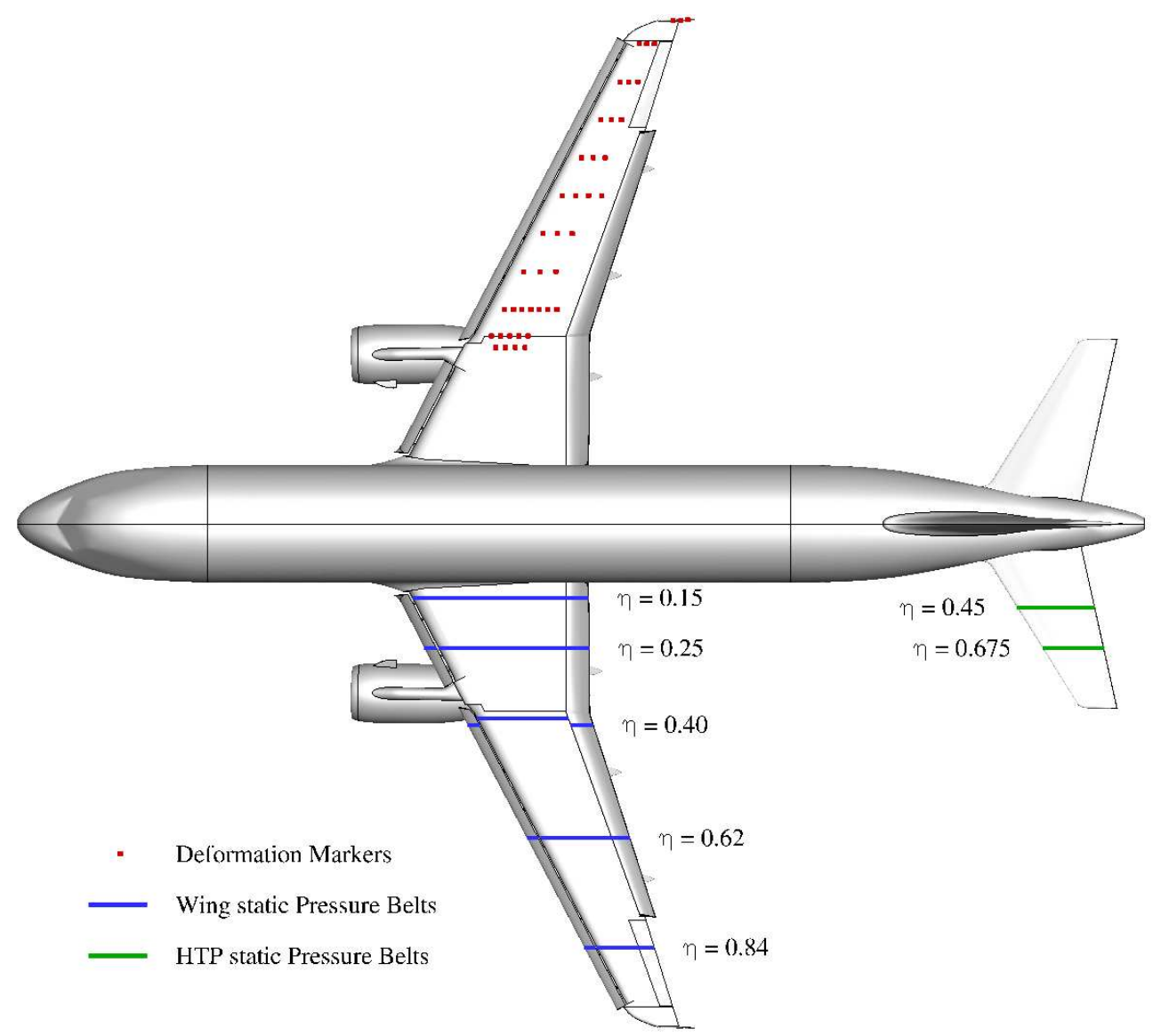

Figure 6. Instrumentation used for validation.

simultaneously. As a result, no pressure distributions on the HTP were available from flight no. 283 and no deformation data was available from flight no. 284. Main selection criteria for the stabilized points listed in Table 1 were based on data quality, including yaw angles equal to or close to zero, constant and symmetric control surface deflections, no spoiler deflections, and low fluctuations over time.

Table 1. Validation Flight Test Cases.

\begin{tabular}{ccccccccc}
$\begin{array}{c}\text { Case } \\
\text { No. }\end{array}$ & $\begin{array}{c}\text { Flight } \\
\text { No. }\end{array}$ & $\begin{array}{c}\alpha \\
{[\mathrm{deg}]}\end{array}$ & $\begin{array}{c}M a \\
{[-]}\end{array}$ & \multicolumn{2}{c}{$h$} & \multicolumn{2}{c}{$m$} \\
\hline 1 & 283 & 17.86 & 0.196 & 2478.3 & 8130.9 & 1882.2 & 67,966 & 149,840 \\
2 & 284 & 18.91 & 0.181 & 2382.2 & 7815.6 & 1743.9 & 67,240 & 148,240
\end{tabular}

\section{Results}

In this section numerical and experimental results for the test cases listed in Table 1 are introduced. Despite being the largest member of DLR's fleet of research aircraft, the A320's moderate size makes it a comparatively stiff structure. Therefore, the observed aeroelastic effects generally remain small, but, given HINVA's ambitious accuracy goals, should still be accounted for. Of particular interest within the scope of these investigations are:

- the role of aeroelastic effects in the simulation of flow phenomena and prediction of flight performance near the point of maximum lift, 
- the impact of slat and flap deformations on overall high-lift performance, and

- the influence of fuselage deformations on trim state and HTP pressure distributions.

\section{Wing Pressure Distribution}

In Figure 7 the chordwise distribution of static pressure coefficient $c_{p}$ in sections no. 1 to no. 4 is plotted for test case no. 1. Results shown include the conventional CFD computation, the FSC simulation on the completely elastic aircraft, and measured data from flight no. 283. Generally, a good correlation between both numerical methods and the experimental data is observed. Minor differences between computed and measured $c_{p}$-distributions are found in the suction peak on the flap upper side in sections no. 1,3 , and 4 . These are possibly due to the simplified inner shroud geometry in the CFD meshes and corresponding alterations in the flow around the flap nose area.
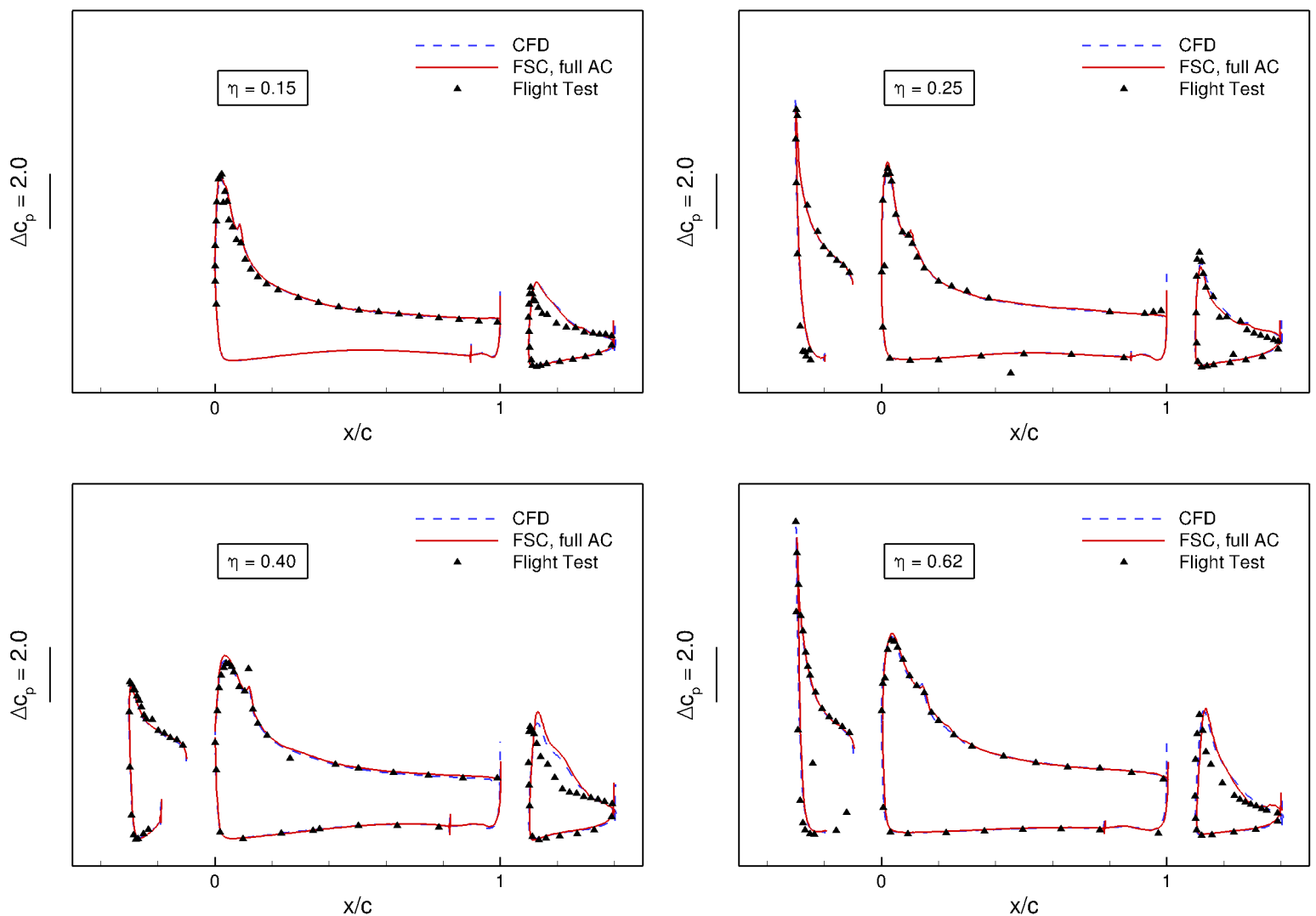

Figure 7. Comparison of chordwise static pressure distributions in four spanwise wing sections for test case no. 1 .

Results for the conventional CFD computation and the FSC simulation do not show significant differences. This is due to the fact that the spanwise wing twist deformation $\varepsilon$ for the aeroelastic equilibrium state computed by the FSC simulation very closely matches the cruise design twist distribution, which is used in the conventional CFD analysis, Figure 9 (b). Apart from the most outboard wing region, i.e. for $\eta>$ 0.85 , deviations between FSC and design twist are always smaller that $0.1 \mathrm{deg}$. As a result, static pressure distributions from both numerical approaches remain extremely small.

\section{Wing Surface Flow Pattern}

On the A320-232 lift breakdown is closely related to the development of vortices emanating from the nacelle strake and the upstream wing/pylon intersection and propagating over the upper wing surface. To judge the influence of aeroelastic effects on the development of these vortices a top view of the nacelle/pylon region including static pressure distribution and surface streamlines is plotted in Figure 8 . 


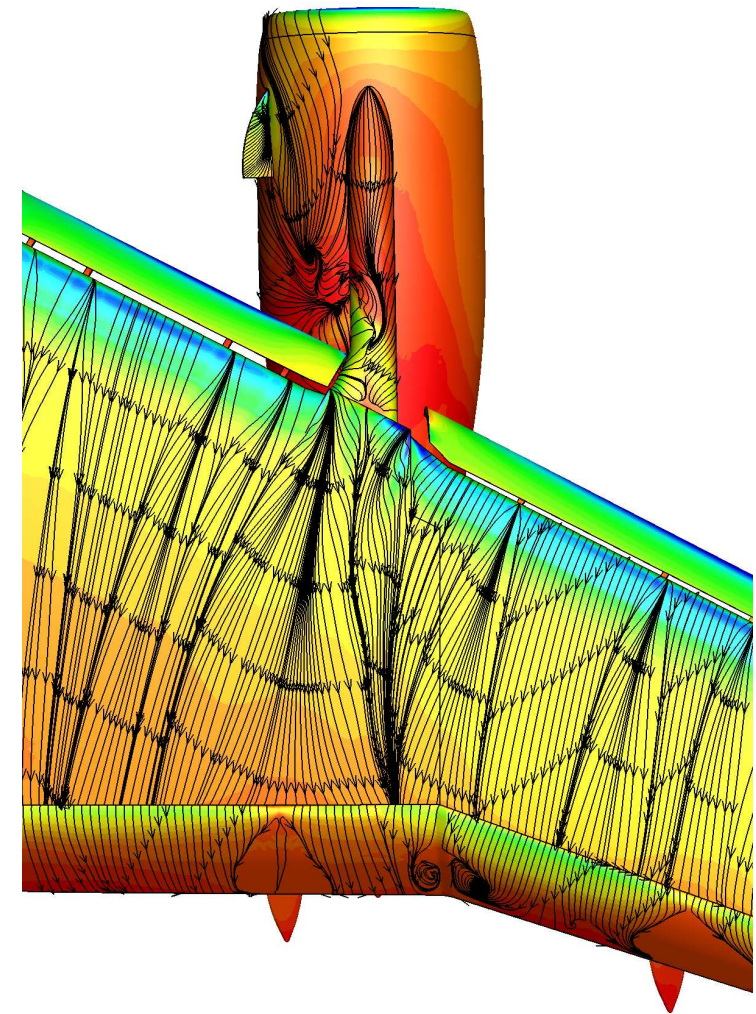

(a) CFD

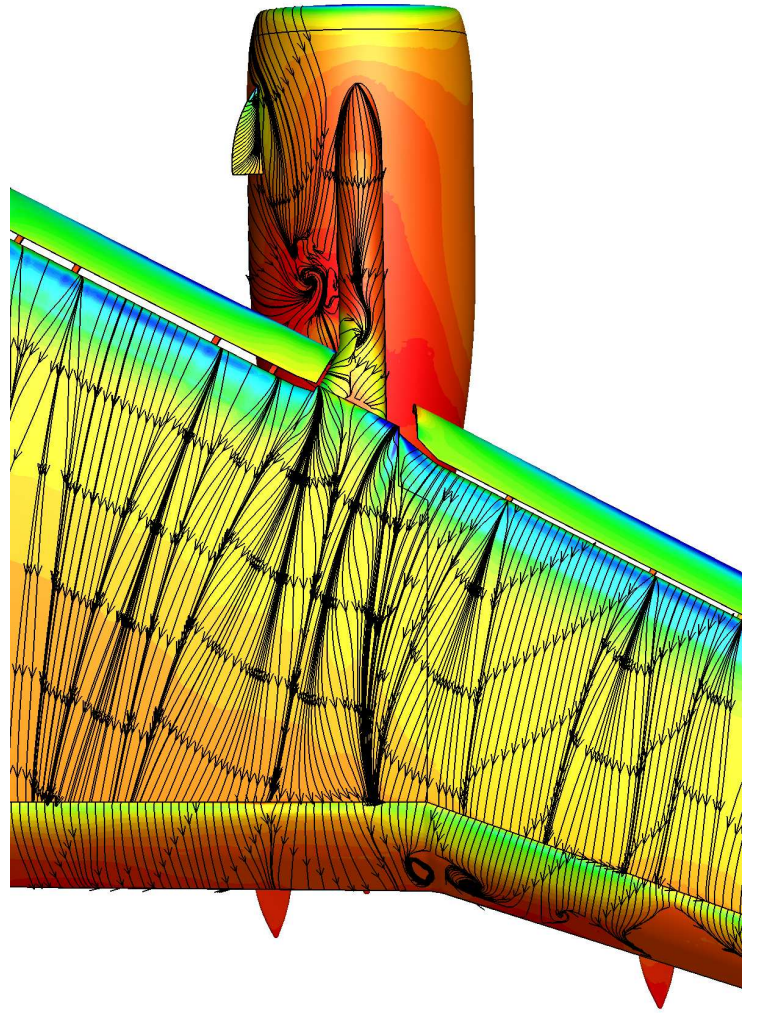

(b) FSC

Figure 8. Surface static pressure distribution and streamlines; test case no. 1.

While the vortex caused by the nacelle strake is barely affected by aeroelastic effects, the vortex system produced by the wing/pylon intersection shows an noticeable reaction to the aircraft's deformation. Besides an influence on the vortex origin on the nacelle upper side and its influence on the surface pressure at the most outboard part of the inboard slat, it can be seen that the vortex traces on the wing's upper side above the engine are clearly shifted outboard in the FSC result. The effect of the shifted vortices is also noticeable when focusing on surface pressure in the aft part of the main wing. Here, a slightly lower pressure level is visible in the FSC simulation result, which is also visible in Figure 7 at $\eta=0.40$. Furthermore the shifted vortices also have an effect on the flow on the flap upper side. The flow separation above the inboard flap track fairing visible in the results of the conventional CFD simulation is not present in the results of the FSC simulation.

\section{Deformation of Wing and High-Lift Devices}

Comparison of FSC twist distribution to measured data indicates a good agreement with $\delta \varepsilon<0.2 \mathrm{deg}$ between $\eta=0.50$ and $\eta=0.875$ and a marginal deviation increase to $\delta \varepsilon \approx 0.3 \mathrm{deg}$ towards the innermost section at $\eta=0.35$ and the wing tip.

Wing bending deformations $w$, Figure 9 (a), show a very good agreement with a maximum deflection difference of $\delta w=16 \mathrm{~mm}(0.63 \mathrm{in})$ at $\eta=0.95$ and a small deviation in slope from $\eta=0.50$ outwards.

The static pressure distributions in Figure 7 already indicate that deformations of the high-lift devices, if at all present, apparently are not relevant aerodynamically. Still, an attempt was made to quantify any existing deflection magnitudes. Figure 10 shows the outlines of wing, slat, and flap at $\eta=0.75$, just outboard of flap track no. 3 for test case no. 1. The jig shape is represented by an orange line, the flight shape is plotted in blue. To eliminate the global bending and twist deformation, the flight shape contour has been shifted and rotated such that its main wing section matches the jig shape. This allows to visualize the relative deflections of slat and flap wrt. the wing. In this test case no significant slat deformation arises, even though the slats carry about $16 \%$ of overall wing lift. The flap incidence angle is increased by approximately 


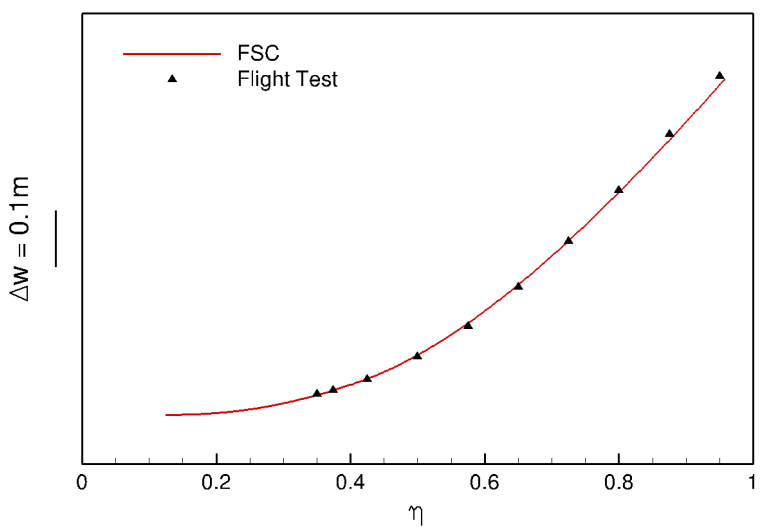

(a) Bending

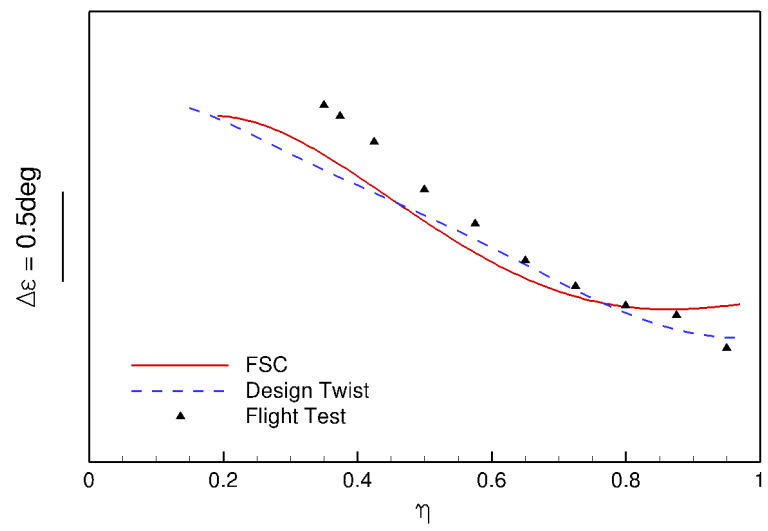

(b) Twist

Figure 9. Comparison of spanwise wing deformations; test case no. 1.

0.25deg. This somewhat unexpected behavior is caused by the strong suction peak near the leading edge and the flap kinematics, which enable a rotation of the flap in the deployed position.

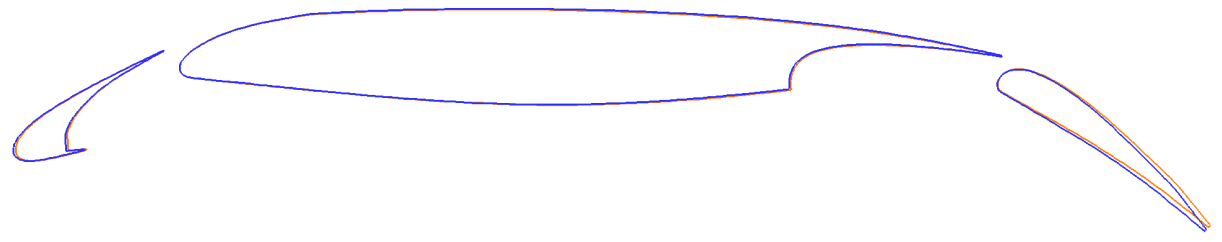

Figure 10. Deformation of high-lift devices (orange: jig shape, blue: flight shape).

\section{Fuselage Deformation and HTP Pressure Distribution}

Preliminary investigations concerning the influence of fuselage deformations on longitudinal trim state and HTP pressure distributions have shown that gravitational forces and aerodynamic forces acting on the horizontal stabilizer lead to a bending deformation of the fuselage tube and a change in the effective HTP incidence angle $i_{H T P}$, Figure 11. Flow around the deformed fuselage and the increased HTP incidence angle create an additional nose-down pitching moment, which requires adjusting $i_{H T P}$ in the order of 0.5 to $1.0 \mathrm{deg}$, depending on angle of attack, to maintain a trimmed flight state, i.e. $C_{M y}=0$, Figure 12, Including the effects of fuselage deformation into the FSC simulations was therefore considered helpful in the comparison of computed HTP pressure distributions to flight test results, where the actual HTP incidence is unknown and therefore constitutes an additional uncertainty.

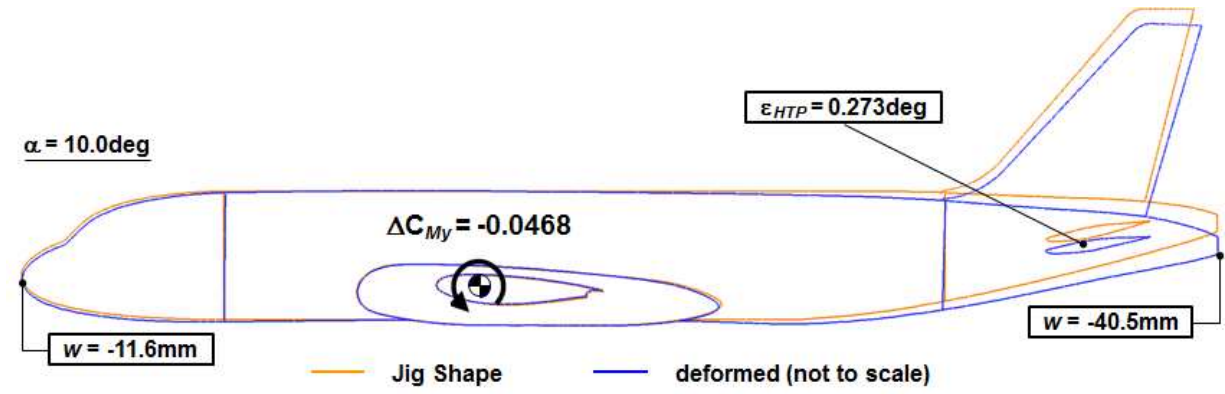

Figure 11. Influence of fuselage deformation on pitching moment. 


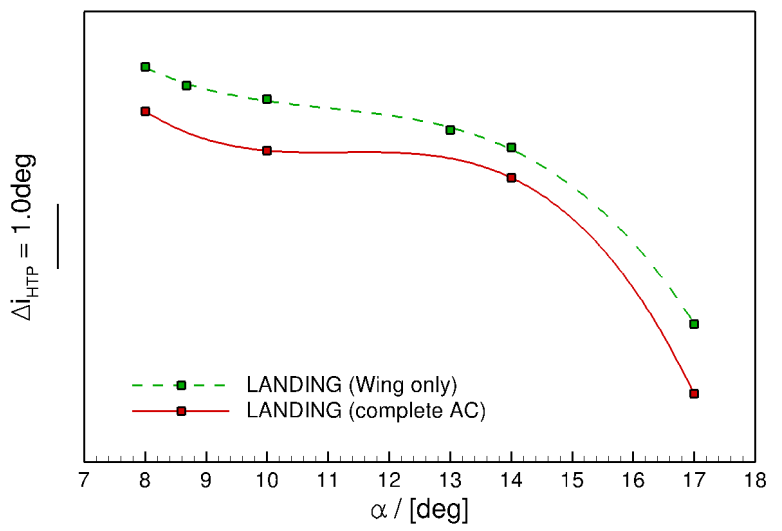

Figure 12. Influence of fuselage deformation on longitudinal trim state.

Figure 13 shows a comparison between chordwise $c_{p}$-distributions for the conventional CFD solution and FSC results at the outboard HTP section. FSC simulations were run without ('Wing only') and with ('full AC') fuselage deformation. Additionally, the configuration with fuselage deformation was run without trimming.

The largest differences occur between the untrimmed and trimmed simulations. The corresponding changes in $i_{H T P}$ range from approximately 0.7 to $1.0 \mathrm{deg}$. Differences between CFD and untrimmed FSC remain relatively small, the untrimmed FSC, however, has a larger nose-down pitching moment due to the deformation of the fuselage tube. Both the trimmed simulations result in virtually indistinguishable pressure distributions but have different HTP incidence angles (cf. Figure 12). The results indicate that the changes in HTP incidence angel due to fuselage deformation, although being very small, have a considerable influence on HTP pressure distribution. For an accurate comparison of numerical results to flight test data the complete aircraft should therefore be considered elastic.

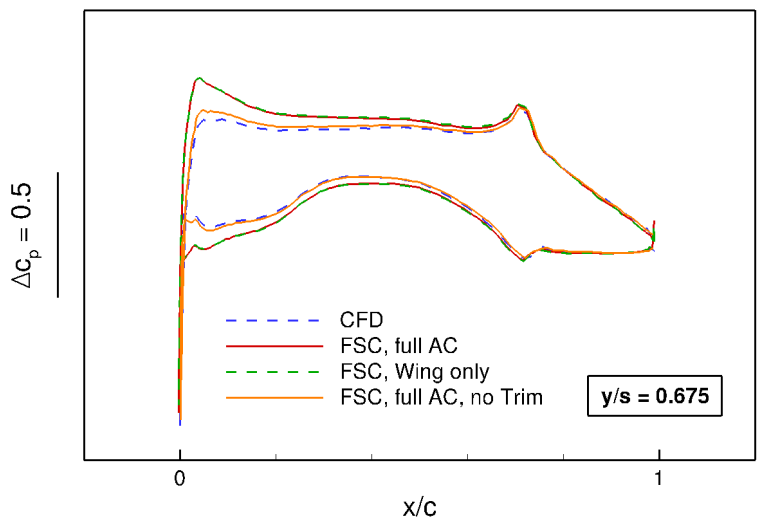

Figure 13. Influence of fuselage deformation on chordwise static pressure coefficient.

Because measured HTP pressures were not available for flight no. 283, the comparison of numerical and experimental data will be based on the second test case, Table 1. In Figure 14 results from the CFD and FSC simulations are compared to measured data. Although deviations between computed and measured $c_{p}$-distributions are generally larger than on the wing, a better correlation of FSC and flight test results is clearly observable, in particular in the outboard section at $\eta=0.675$. This confirms that including fuselage deformation yields an improved correlation between FSC simulation results and flight test data. 

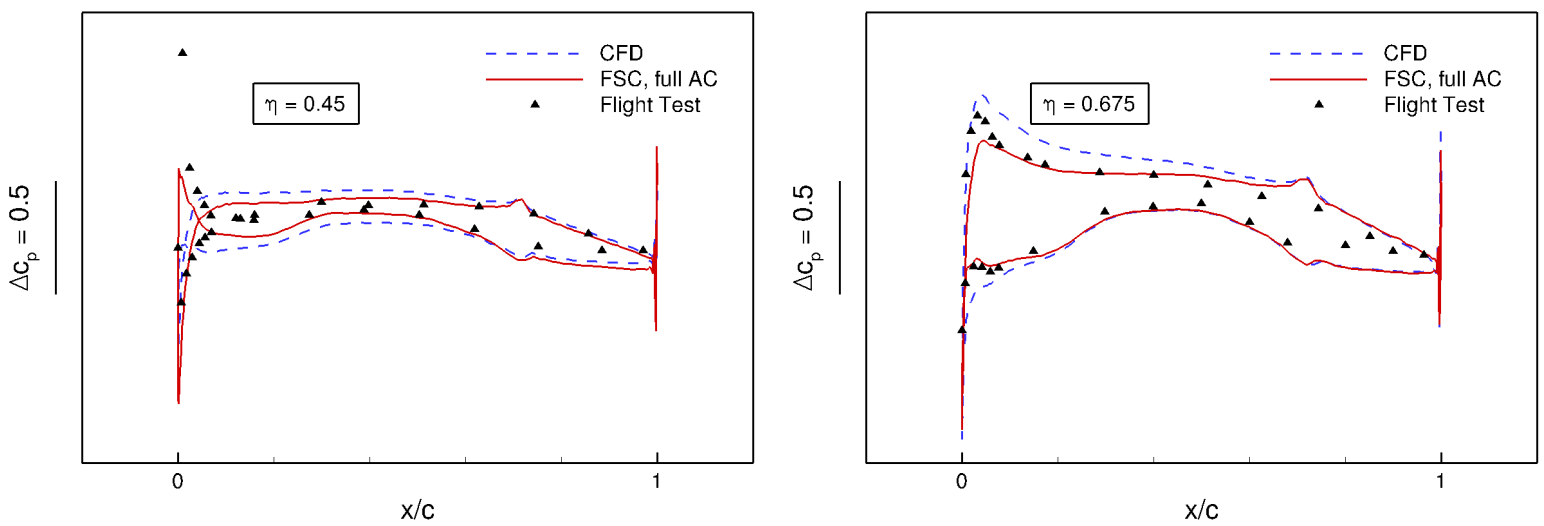

Figure 14. Comparison of chordwise static pressure distributions on HTP; test case no. 2 .

\section{Conclusions}

The influence of aeroelastic effects on the aerodynamic performance of DLR's Airbus A320-232 ATRA research aircraft in high-lift configuration has been investigated. Fluid-structure-coupled simulations were performed and compared to both conventional CFD analyses and experimental data from HINVA's first flight test campaign in July 2012. Aeroelastic effects were found to be generally small, owing to the A320's comparatively rigid structure. In particular, no significant influence of wing deformation on chordwise static pressure distributions was observed. However, differences in the development of the inboard wing vortex system, i.e. in the region where lift breakdown occurs, were identified. The influence of fuselage deformations on HTP pressure distributions and trim state was investigated. Here, deviations between conventional CFD and FSC simulations were considerably larger than on the wing, with the FSC results showing the better correlation to flight test data.

\section{Acknowledgments}

The joint research project HINVA is funded by the German Federal Ministry of Economics and Technology within the fourth Aeronautical Research Program LuFo IV. Experimental deformation data was kindly provided by Ralf Meyer, Institute of Aerodynamics and Flow Technology, Dept. Experimental Methods.

\section{References}

${ }^{1}$ Rudnik, R., Reckzeh, D., and Quest, J., "HINVA - High lift INflight VAlidation - Project Overview and Status," Paper 2012-0106, AIAA, 2012.

${ }^{2}$ Heinrich, R., Wild, J., Streit, T., and Nagel, B., "Steady Fluid-Structure Coupling for Transport Aircraft," ONERA-DLR Aerospace Symposium, Oct. 2006.

${ }^{3}$ Gerhold, T., "Overview of the Hybrid RANS Code TAU," MEGAFLOW, edited by N. Kroll and J. Fassbender, Vol. 89 of Notes on Numerical Fluid Mechanics and Multidisciplinary Design, Springer, 2005, pp. 81-92.

${ }^{4}$ MSC Software Corporation, "Product Information," [online web site], https://www.mscsoftware.com/product/mscnastran, 2013

${ }^{5}$ Bier, N., Rohlmann, D., and Rudnik, R., "Numerical Maximum Lift Predictions of a Realistic Commercial Aircraft in Landing Configuration," AIAA Paper 2012-0279, January 2012.

${ }^{6}$ CentaurSoft, "Centaur Hybrid Grid Generation System," [online web site], http://www.centaursoft.com, 2013.

${ }^{7}$ Jameson, A., Schmidt, W., and Turkel, E., "Numerical Solution of the Euler Equations by Finite Volume Methods using Runge-Kutta Time Stepping Schemes," AIAA Paper 81-1259, Jan. 1981.

${ }^{8}$ Swanson, R. C. and Turkel, E., "On Central Differences and Upwind Schemes." Journal of Computational Physics, Vol. 101, pp., 1992. 1992.

${ }^{9}$ Spalart, P. and Allmaras, S., "A One-Equation Turbulence Model for Aerodynamic Flows," AIAA Paper 1992-0439,

${ }^{10}$ MSC Nastran 2007r1 Quick Reference Guide, MSC Software Corporation, 2007. 